\title{
Urinary neutrophil gelatinase-associated lipocalin: a marker of urinary tract infection among febrile children
}

\author{
Ji Hyun Moon, MD ${ }^{1}$, Kee Hwan Yoo, MD, PhD ${ }^{1}$, Hyung Eun Yim, MD, PhD ${ }^{2}$ \\ ${ }^{1}$ Department of Pediatrics, Korea University Guro Hospital, Seoul, Korea; ${ }^{2}$ Department of Pediatrics, Korea University Ansan Hospital, Ansan, Korea
}

Background: Neutrophil gelatinase-associated lipocalin (NGAL) has emerged as a valuable biomarker of urinary tract infection (UTI) in children.

Purpose: This study aimed to compare the diagnostic accuracy of urinary NGAL (uNGAL) with those of serum C-reactive protein (CRP) and white blood cell (WBC) count for predicting UTI and acute pyelonephritis (APN) in febrile children.

Methods: The medical charts of children undergoing uNGAL measurements between November 2017 and August 2019 were retrospectively reviewed. Patients with a suspected or diagnosed UTIs were included. The diagnostic accuracies of uNGAL, serum CRP, and WBC count for detecting UTI and APN were investigated. Independent predictors of UTI and APN were investigated using multivariable logistic regression analyses.

Results: A total of 321 children were enrolled in this study. The uNGAL levels were higher in the UTI group $(n=157)$ than in the non-UTI group $(n=164)(P<0.05)$. Among children with a UTI, uNGAL levels were higher in the APN group $(n=70)$ than, the non-APN group $(n=87)(P<0.05)$. In the multivariate analysis, uNGAL was independently associated with UTI and APN (both $P<0.05$ ). Serum CRP and WBC count were not correlated with the presence of UTI and APN. Receiver operating curve analyses showed that the uNGAL level had the highest area under the curve (AUC) for predicting UTI and APN, respectively (AUC, uNGAL vs. CRP vs. WBC count, 0.860 vs. 0.608 vs. 0.669 for UTI; 0.780 vs. 0.680 vs. 0.639 for APN, all $P<0.05$, respectively). The predictive values and likelihood ratios of uNGAL were superior to those of serum CRP and WBC count for detecting UTI and APN at each cutoff level.

Conclusion: UNGAL may be more useful than serum CRP and WBC count for identifying and assessing UTI in febrile children.

Key words: Bacterial infections, Lipocalin-2, Pyelonephritis

\section{Key message}

Question: Reliably diagnosing urinary tract infection (UTI) in febrile children is often difficult. Can urinary neutrophil gelatinase-associated lipocalin (uNGAL) better predict UTI and acute pyelonephritis (APN) than serum C-reactive protein (CRP) and white blood cell (WBC) count?

Finding: UNGAL better facilitated the prediction of UTI and APN, than serum CRP and WBC counts in febrile children.

Meaning: uNGAL is a suitable biomarker for UTI and APN in febrile children.

\section{Introduction}

Urinary tract infection (UTI) is the one of the most common bacterial infections in infants and children, especially younger than three months, with a prevalence of $7 \% .{ }^{1,2)}$ UTI affects nearly $2 \%$ of boys and $8 \%$ of girls in the first six years of life. ${ }^{3)}$ It should be examined carefully because of its potential for later development of chronic kidney disease (CKD), such as renal scarring. ${ }^{4)}$ Especially, vesicoureteral reflux (VUR) has been considered the most important risk factor for post-UTI renal scar formation in children. ${ }^{5)}$ Patients with suspected UTIs can be screened via urinalysis (UA) and urine culture tests. ${ }^{2}$ Since the urine culture result is not available for 24-48 hours of incubation, a screening UA must be performed for a presumed diagnosis of UTI while the results of the urine culture are displayed. However, the sensitivity and specificity of UA for the diagnosis of UTI vary with age, race, and sex.,6,7) Additionally, urine cultures often give false-positive results from contamination, and if a clinician considers a negative UA result as sufficient evidence for non-UTI, urine culture exam is not obtained, and then there is the probability of missing a UTI. A false negative finding may be also displayed if the patients took an antibiotic before the UA. Also, obtaining a urine sample from infants and toddlers who do not control voiding is difficult. Thus, novel biomarkers to aid UTI diagnosis and to determine the severity of UTI have been investigated in children.

As one of the useful, noninvasive, and convenient biomarkers, neutrophil gelatinase-associated lipocalin (NGAL) is an emerg. ing indicator for diagnosing UTI. ${ }^{8)}$ NGAL, a member of the lipocalin superfamily, is a protein present in neutrophils and

Corresponding author: Hyung Eun Yim, MD, PhD. Department of Pediatrics, Korea University Ansan Hospital, 123 Jeokgeum-ro, Danwon-gu, Ansan 15355, Korea 凶Email: he-yim@hanmail.net, https://orcid.org/0000-0001-9805-9278

Received: 3 July, 2020, Revised: 9 September, 2020, Accepted: 22 September, 2020

This is an open-access article distributed under the terms of the Creative Commons Attribution Non-Commercial License (http://creativecommons.org/licenses/bync/4.0/) which permits unrestricted non-commercial use, distribution, and reproduction in any medium, provided the original work is properly cited.

Copyright (c) 2021 by The Korean Pediatric Society 


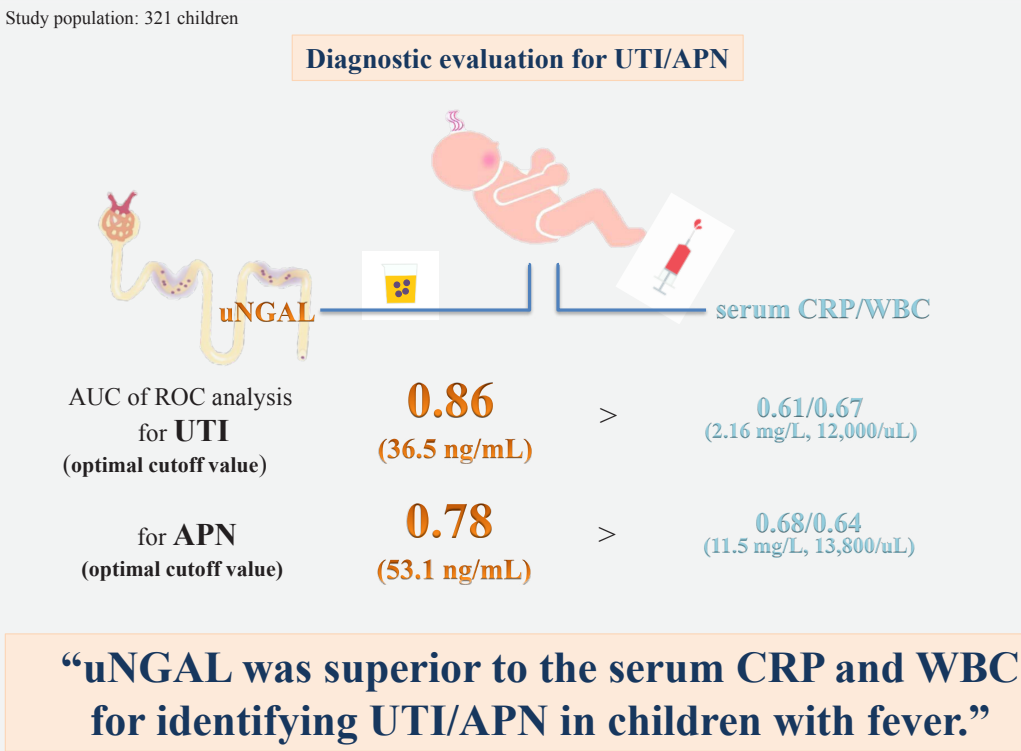

"UNGAL was superior to the serum CRP and WBC for identifying UTI/APN in children with fever."

UTI; urinary tract infection, APN; acute pyelonephritis, uNGAL; urinary neutrophil gelatinase-associated lipocalin

\section{Graphical abstract}

many other human tissues, including $\alpha$-intercalated cells in the collecting duct of the kidney. ${ }^{9,10)}$ NGAL is considered a com. ponent of the innate immune system and inhibits bacterial growth. ${ }^{9)}$ The NGAL expression is significantly induced in injured epithelial cells, including the kidney. ${ }^{11)}$ The NGAL levels in urine and serum are low under normal conditions, but increases rapidly in response to cell damage especially with gram-negative UTI. ${ }^{8,912)}$ Thus, the NGAL has great potential for early diagnosis of UTI. There are 2 forms of the NGAL in the biomarker literature: plasma NGAL (pNGAL) and urine NGAL (uNGAL). pNGAL was secreted by neutrophils as a result of systemic inflammations, and UNGAL is specific for damage to the genitourinary epithelium. ${ }^{13)}$ The uNGAL is significantly elevated with gram-negative UTIs in animal studies. ${ }^{14,15)}$ NGALdeficient mice were more susceptible to bacterial infections with Escherichia coli and died with sepsis. ${ }^{14)}$ Similarly, there are many studies that uNGAL is a novel, sensitive marker for acute kidney injury (AKI) as well as for UTI diagnosis. ${ }^{16,17)}$ However, few studies $^{10,18,19)}$ have compared UNGAL and other biomarkers for predicting UTI and acute pyelonephritis (APN).

Recently, we showed that pNGAL could be a sensitive predictor for identifying APN and monitoring the treatment response of pediatric UTI. The pNGAL was more useful than serum procalcitonin (PCT), C-reactive protein (CRP), and white blood cell (WBC) levels for identifying APN in children with febrile UTIs. ${ }^{20)}$ In this study, we compared the uNGAL and serum CRP and WBC to assess if uNGAL could also be useful in predicting UTI and APN beyond the serum CRP and WBC in children with fever. We investigated the diagnostic accuracy of uNGAL for UTI and APN in children with fever.

\section{Methods}

\section{Study population}

This retrospective study involved children with fever undergoing uNGAL measurements at our single tertiary care center between November 2017 and August 2019. All children aged 1 month to 18 years presenting with fever $\left(\geq 38.0^{\circ} \mathrm{C}\right)$ underwent uNGAL test to rule out UTI when the cause of the fever was not determined at first. Patients with febrile UTIs were defined by fever $\left(\geq 38.0^{\circ} \mathrm{C}\right)$, pyuria ( $\geq 5 \mathrm{WBCs} /$ high power field), and a positive urine culture with a pure growth of $>50,000$ organisms/ $\mathrm{mL}$ on a catheter or midstream urine specimen. Patients with previous UTIs and congenital urinary tract anomalies, AKI, underlying CKD, were excluded. For diagnosing UTI, collection of urine specimens was obtained via ureteral catheterization in nontoilet-trained children or midstream clean catch in toilettrained patients. The Institutional Review Board (IRB) of the Korea University Guro Hospital approved this retrospective study (IRB number: K2019-0819-002), and the need for informed consent was waived.

\section{Laboratory and radiological assessments}

In all patients undergoing uNGAL measurements, urine and blood samples were obtained before treatment including antibiotic administration. The uNGAL measurements were performed using the commercial chemiluminescentmicroparticle immunoassay assay (ARCHITECT Urine NGAL assay, Abbott Diagnostics, AbbottPark, IL, USA). The ARCHITECT urine NGAL assays are used to detect the monomeric form of NGAL in urine predominantly. In children diagnosed with a first febrile UTI, renal sonography, and technetium-99m-labeled dimercaptosuccinic acid (DMSA) renal scintigraphy was performed on 
admission. Patients with APN findings on DMSA scan underwent voiding cystourethrogram (VCUG) before hospital discharge. Since children with APN are more likely to have VUR, VCUG was carried out in the APN group according to the top-down approach for UTI screening. ${ }^{21,22)}$ Among children without APN, VCUG was performed in cases with a suspicion of VUR on renal sonography. Hydronephrosis was defined based on the Society for Fetal Urology classification ${ }^{23)}$ and APN was diagnosed when the DMSA renal scan showed a focal, multifocal or diffuse reduction or lack of uptake. The DMSA scans were performed at the time of diagnosis of UTI. Patients undergoing uNGAL measurements were divided into 2 groups (UTI and non-UTI) as defined previously. Patients with UTIs were additionally subdivided into APN and non-APN groups, according to the results of DMSA scan. Clinical and laboratory findings were compared between the UTI and the non-UTI group and between the APN and the non-APN group. The diagnostic accuracy of the UNGAL, CRP, and WBC for detecting UTI and APN was investigated.

\section{Statistical analysis}

We used SPSS ver. 18.0 (SPSS Inc., Chicago, IL, USA) and the MedCalc Statistical Software ver. 18 (MedCalc Software bvba, Ostend, Belgium). The categorical variables were compared with the chi-square test and the continuous variables were compared using the Student $t$ test and the Mann-Whitney $U$ test. The continuous variables that did not follow a normal distribution were compared with the Mann-Whitney $U$ test. The odds ratios (ORs) were calculated by logistic regression analyses to identify the risk factors for the development of UTI and APN. To determine independent risk factors, the variables related to UTI and APN $(P<0.05)$ in the univariable analyses were then tested in the multivariable logistic regression models, respectively. The receiver operating characteristic (ROC) analysis was performed, and the areas under the curves (AUCs) of the UNGAL, CRP, WBC for detecting UTI and APN were calculated. The AUCs of the uNGAL, CRP, WBC were then compared using the method described by Hanley and McNeil. ${ }^{24)}$ Sensitivity, specificity, positive predictive value (PPV), negative predictive value (NPV), positive likelihood ratio (PLR), and negative likelihood ratio (NLR) were calculated for each biomarker. The data were displayed as the mean ( \pm standard deviation) or median (interquartile range) of continuous variables and percentage of categorical variables. Statistical significance was set at $P<0.05$.

\section{Results}

\section{Patient characteristics}

The flow diagram for the patients group is summarized Fig. 1. uNGAL concentration was measured in 329 patients from November 2017 to August 2019. Among them, 8 patients with a history of UTIs, AKI, underlying CKD, and congenital urinary tract anomalies were excluded. Of the enrolled 321 children, 157 patients (48.9\%) were included in the UTI group and 164 patients $(51.1 \%)$ were in the non-UTI group. The non-UTI group comprised viral upper respiratory infection $(n=63,38.4 \%)$, viral acute gastroenteritis $(n=36,22.0 \%)$, viral pneumonia $(n=31,18.9 \%)$, cervical lymphadenitis $(n=8$, 4.8\%), and others $(n=26,15.9 \%)$. Among 157 children with UTI, 70 patients (44.6\%) were included in the APN group and 87 patients (55.4\%) were in the non-APN group.

\section{Clinical and laboratory findings}

In the UTI group, the mean age was lower and fever duration was shorter, compared with the non-UTI group (both $P<0.05$ ). Patients with UTI had higher levels of the serum WBC and CRP, and uNGAL at admission, compared with children with nonUTI $(P<0.05)$. Also, fever duration in patients with UTI was shorter than in non-UTI patients $(P<0.05)$. The hemoglobin concentration and serum creatinine levels were lower in the UTI group $(P<0.05)$. Among the UTI patients, mean age, sex distribution, and fever duration did not differ between the APN group and the non-APN group. The 2 groups showed no differences in serum WBC, hemoglobin, and creatinine levels. In the APN group, the serum CRP and uNGAL levels were higher than in the non-APN group $(P<0.05)$. Besides, the incidence of VUR was higher in the APN group $(P<0.05)$. However, the incidence of hydronephrosis did not differ between the 2 groups (Table 1).

\section{Univariable and multivariable logistic regression analyses}

The univariable analysis identified several clinical variables associated with the presence of UTI and APN as shown in Tables 2 and 3. Age, sex, fever duration, serum WBC, hemoglobin, and uNGAL were associated with the presence of UTI (all $P<0.05)$. However, in a multivariable analysis for UTI, age (odds ratio [OR], 0.49; $95 \%$ confidence interval [CI], 0.32-0.75, $P=0.001$ ) and uNGAL (OR, 1.13; 95\% CI, 1.08-1.18; $P=0.001)$ levels independently predicted UTI. We determined the possible

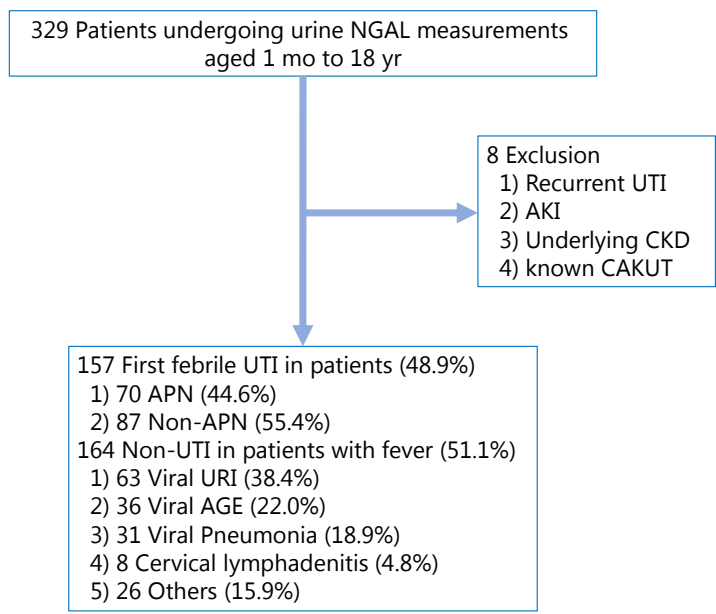

Fig. 1. Flow diagram. Study population. NGAL, neutrophil gelatinaseassociated lipocalin; UTI, urinary tract infection; AKI, acute kidney injury; CKD, chronic kidney disease; CAKUT, congenital anomalies of the kidney and urinary tract; APN, acute pyelonephritis. 
Table 1. Clinical and laboratory findings of all patients and UTI patients

\begin{tabular}{|c|c|c|c|c|c|c|}
\hline Variable & Non-UTI $(n=164)$ & UTI $(n=157)$ & $P$ value & Non-APN $(n=87)$ & APN $(n=70)$ & $P$ value \\
\hline Age (mo) & $60.3 \pm 62.3$ & $16.8 \pm 33.7$ & $<0.001^{\mathrm{a})}$ & $21.6 \pm 39.5$ & $10.9 \pm 24.0$ & $0.319^{a)}$ \\
\hline Female sex & $82 / 164(50.0)$ & $57 / 157(36.3)$ & $0.013^{\mathrm{a})}$ & $31 / 87(35.6)$ & $26 / 70(37.1)$ & $0.845^{b)}$ \\
\hline Fever duration, $>72 \mathrm{hr}$ & $41 / 164$ (25.0) & $18 / 157(11.5)$ & $0.002^{b)}$ & $9 / 87(10.3)$ & $9 / 70(12.8)$ & $0.624^{b)}$ \\
\hline WBC $(\mu \mathrm{L})$ & $11,020 \pm 6,573$ & $13,664 \pm 5,693$ & $<0.001^{\mathrm{a})}$ & $13,088 \pm 5,335$ & $14,381 \pm 6,071$ & $0.213^{\mathrm{a})}$ \\
\hline $\mathrm{Hb}(\mathrm{g} / \mathrm{dL})$ & $12.1 \pm 1.3$ & $11.2 \pm 1.2$ & $<0.001^{c)}$ & $11.4 \pm 1.3$ & $11.1 \pm 1.2$ & $0.172^{c)}$ \\
\hline $\mathrm{CRP}(\mathrm{mg} / \mathrm{L})$ & $29.1 \pm 45.4$ & $33.3 \pm 40.5$ & $0.001^{a)}$ & $23.7 \pm 32.6$ & $45.3 \pm 46.0$ & $0.001^{\mathrm{a})}$ \\
\hline Creatinine (mg/dL) & $0.4 \pm 0.2$ & $0.2 \pm 0.1$ & $0.001^{a)}$ & $0.3 \pm 0.1$ & $0.2 \pm 0.1$ & $0.085^{a)}$ \\
\hline uNGAL (ng/mL) & $31.6 \pm 63.1$ & $240.9 \pm 292.4$ & $<0.001^{\mathrm{a})}$ & $200.1 \pm 270.7$ & $291.6 \pm 311.8$ & $0.014^{\mathrm{a})}$ \\
\hline Hydronephrosis & - & - & - & $60 / 87(68.9)$ & $58 / 70(82.8)$ & $0.076^{b)}$ \\
\hline VUR & - & - & - & $0 / 6(0)$ & $17 / 69(24.6)$ & $0.001^{b)}$ \\
\hline
\end{tabular}

Values are presented as mean \pm standard deviation or number (\%).

UTI, urinary tract infection; APN, acute pyelonephritis; WBC, white blood cell; Hb, hemoglobin; CRP, C-reative protein; uNGAL, urinary neutrophil gelatinaseassociated lipocalin; VUR, vesicoureteral reflux.

a) Mann-Whitney $U$ test. ${ }^{\text {b) }}$ Chi-square test. ${ }^{\mathrm{c})}$ Student $t$ test.

Boldface indicates a statistically significant difference with $P<0.05$.

Table 2. Univariable and multivariable logistic regression analyses for UTI in all patients

\begin{tabular}{|c|c|c|c|c|}
\hline \multirow{2}{*}{ Variable } & \multicolumn{2}{|c|}{ Univariable } & \multicolumn{2}{|c|}{ Multivariable } \\
\hline & Odds ratio $(95 \% \mathrm{Cl})$ & $P$ value & Odds ratio $(95 \% \mathrm{Cl})$ & $P$ value \\
\hline Age (mo) & $0.31(0.21-0.46)$ & 0.001 & $0.49(0.32-0.75)$ & 0.001 \\
\hline Female sex & $0.57(0.36-0.89)$ & 0.014 & $0.84(0.47-1.48)$ & 0.534 \\
\hline Fever duration, $>72 \mathrm{hr}$ & $0.39(0.21-0.71)$ & 0.002 & $0.78(0.54-1.18)$ & 0.364 \\
\hline$W_{B C}{ }^{a)}$ & $1.11(1.06-1.16)$ & 0.001 & $1.02(0.97-1.08)$ & 0.395 \\
\hline $\mathrm{CRP}^{\mathrm{a})}$ & $1.02(0.97-1.08)$ & 0.383 & - & - \\
\hline $\mathrm{UNGAL}^{\mathrm{a})}$ & $1.16(1.10-1.21)$ & 0.001 & $1.13(1.08-1.18)$ & 0.001 \\
\hline $\mathrm{Hb}$ & $0.59(0.48-0.71)$ & 0.001 & 0.90 (0.69-1.18) & 0.453 \\
\hline
\end{tabular}

UTI, urinary tract infection; $\mathrm{Cl}$, confidence interval; WBC, white blood cell; CRP, C-reactive protein; UNGAL, urinary neutrophil gelatinase-associated lipocalin; $\mathrm{Hb}$, hemoglobin.

a) Odds ratio represents the incremental odds of UTI for every unit increase of one standard deviation in age, or 1,000 cells per $\mu \mathrm{L}$ in WBC, or $10 \mathrm{mg}$ per $\mathrm{L}$ in CRP, 10 ng per $\mathrm{mL}$ in UNGAL.

Boldface indicates a statistically significant difference with $P<0.05$.

Table 3. Univariable and multivariable logistic regression analyses for APN

\begin{tabular}{|c|c|c|c|c|}
\hline \multirow{2}{*}{ Variable } & \multicolumn{2}{|c|}{ Univariable } & \multicolumn{2}{|c|}{ Multivariable } \\
\hline & Odds ratio $(95 \% \mathrm{Cl})$ & $P$ value & Odds ratio $(95 \% \mathrm{Cl})$ & $P$ value \\
\hline Age (mo) & $0.96(0.27-1.04)$ & 0.811 & - & - \\
\hline Female sex & $0.79(0.56-2.05)$ & 0.231 & - & - \\
\hline Fever duration, $>72 \mathrm{hr}$ & $0.78(0.48-3.42)$ & 0.150 & - & - \\
\hline WBC $^{a)}$ & $1.04(0.98-1.10)$ & 0.160 & - & - \\
\hline $\mathrm{CRP}^{\mathrm{a})}$ & $1.16(1.06-1.28)$ & 0.002 & $1.01(0.99-1.02)$ & 0.306 \\
\hline uNGAL a) & $1.12(1.08-1.15)$ & 0.023 & $1.15(1.04-1.27)$ & 0.038 \\
\hline $\mathrm{Hb}$ & $0.83(0.64-1.08)$ & 0.163 & - & - \\
\hline
\end{tabular}

APN, acute pyelonephritis; Cl, confidence interval; WBC, white blood cell; CRP, C-reactive protein; UNGAL, urinary neutrophil gelatinase-associated lipocalin; $\mathrm{Hb}$, hemoglobin.

a) Odds ratio represents the incremental odds of UTI for every unit increase of one standard deviation in age, or 1,000 cells per $\mu \mathrm{L}$ in WBC, or $10 \mathrm{mg}$ per $\mathrm{L}$ in CRP, 10 ng per $\mathrm{mL}$ in UNGAL.

Boldface indicates a statistically significant difference with $P<0.05$.

multicollinearity between the 2 variables of age and uNGAL. No correlation was found between the 2 variables $(1<$ variance inflation factor $<10$ ). For the APN, the univariable analyses showed that the serum CRP and UNGAL were related with the presence of the APN (all $P<0.05$ ). In a multivariable analysis for the APN, elevated uNGAL was the only independent predictor of the presence of the APN (OR, 1.15; 95\% Cl, 1.04-1.27;
$P=0.038)$.

\section{Diagnostic performance of UNGAL and serum CRP and WBC}

We performed ROC analyses to define the diagnostic efficacy of uNGAL and serum CRP and WBC for the detection of UTI and APN. For the UTI, the AUC value of UNGAL is $0.86(95 \%$ CI, 0.82-0.90), followed by the serum WBC of 0.67 (95\% 
CI, 0.62-0.72) and the CRP of 0.61 (95\% CI, 0.55-0.66) (all $P<0.001$ ). In a pairwise comparison, the AUC value of uNGAL for UTI was significantly higher than that of the CRP and that of the WBC count (both $P<0.05$ ). For APN, the AUC value of uNGAL is 0.78 (95\% CI, 0.73-0.82), followed by the CRP of 0.68 (95\% CI, 0.63-0.73), WBC of 0.64 (95\% CI, $0.58-0.69$ ) (all $P<0.001$ ). The pairwise comparison analyses showed that the AUC of uNGAL level for UTI and APN was the highest, compared with that of the CRP and that of the WBC (all $P<0.05$ ). The optimal cutoff levels for detecting UTI with uNGAL, CRP, and WBC were $36.5 \mathrm{ng} / \mathrm{mL}, 2.16 \mathrm{mg} / \mathrm{L}$, and $12,000 / \mu \mathrm{L}$, respectively. At the best cutoff value of $36.5 \mathrm{ng} /$ $\mathrm{mL}$ of uNGAL for UTI, sensitivity and specificity were $81.5 \%$ and $79.3 \%$, respectively. Among all examined biomarkers, the uNGAL showed the highest PPV (79.0\%), NPV (81.8\%), and the PLR (3.93; $95 \%$ CI, 2.9-5.4) and the lowest NLR $(0.23$; 95 $\% \mathrm{CI}, 0.2-0.3$ ) for detecting UTI. For identifying the APN, the best cutoff levels with the uNGAL, CRP, and WBC were $53.1 \mathrm{ng} /$
$\mathrm{mL}, 11.5 \mathrm{mg} / \mathrm{L}$, and 13,800/uL respectively. At the cutoff level of $53.1 \mathrm{ng} / \mathrm{mL}$ of uNGAL for the APN, sensitivity and specificity were $81.7 \%$ and $67.2 \%$, respectively. Additionally, the uNGAL showed the greatest PPV (41.4\%), NPV (92.8\%), and the PLR (2.49; 95\% CI, 2.0-3.1) and the lowest NLR (0.27; 95\% CI, $0.2-0.4$ ) for detecting APN among the examined biomarkers (Table 4) (Fig. 2).

\section{Discussion}

This study compared the diagnostic accuracy of uNGAL with the serum CRP and WBC for predicting UTI and APN in children with fever. The results demonstrate that the uNGAL level could be useful in predicting UTI and APN beyond the serum CRP and WBC. Using the logistic regression analyses, we found that the elevated uNGAL level was an independent predictor of UTI and APN in children with fever. For predicting
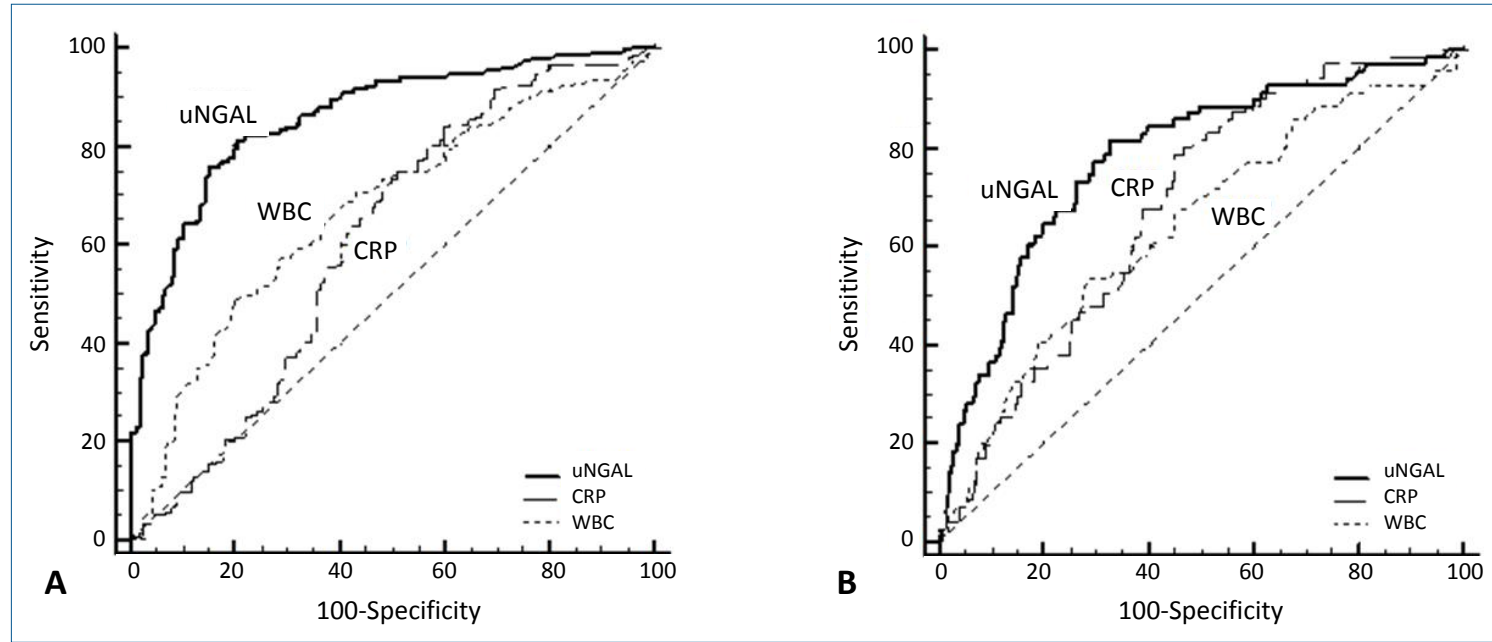

Fig. 2. Receiver operating characteristic analysis of urine neutrophil gelatinase-associated lipocalin (UNGAL), serum C-reactive protein (CRP) levels, and white blood cell (WBC) count for predicting urinary tract infection (UTI) (A) and acute pyelonephritis (APN) (B). (A) $P$ values from each pairwise comparison for UTI: UNGAL to WBC count, $P<0.0001$; uNGAL to CRP, $P<0.0001$; WBC count to $\mathrm{CRP}, P=0.097$. (B) $P$ values from each pairwise comparison for APN: UNGAL to WBC count, $P=0.0005$; UNGAL to CRP, $P=0.004$; WBC count to $C R P, P=0.320$.

Table 4. Sensitivity, specificity, PPV, NPV, PLR, and NLR of WBC, CRP, and UNGAL measurements for predicting UTI, APN

\begin{tabular}{|c|c|c|c|c|c|c|}
\hline \multirow{2}{*}{ Variable } & \multicolumn{3}{|c|}{ UTI } & \multicolumn{3}{|c|}{ APN } \\
\hline & uNGAL (ng/mL) & $\mathrm{CRP}(\mathrm{mg} / \mathrm{L})$ & WBC $(\mu \mathrm{L})$ & uNGAL (ng/mL) & $\mathrm{CRP}(\mathrm{mg} / \mathrm{L})$ & WBC $(\mu \mathrm{L})$ \\
\hline Criterion & $>36.5$ & $>2.16$ & $>12,000$ & $>53.1$ & $>11.5$ & $>13,800$ \\
\hline Sensitivity & 81.5 & 84.1 & 49.0 & 81.7 & 78.9 & 53.5 \\
\hline Specificity & 79.3 & 40.2 & 79.9 & 67.2 & 55.2 & 71.6 \\
\hline PLR (95\% Cl) & $3.90(2.9-5.4)$ & $1.10(1.2-1.6)$ & $2.44(1.7-3.4)$ & $2.49(2.0-3.1)$ & $1.76(1.5-2.1)$ & $1.88(1.4-2.5)$ \\
\hline NLR $(95 \% \mathrm{CI})$ & $0.23(0.2-0.3)$ & $0.40(0.3-0.6)$ & $0.64(0.5-0.8)$ & $0.27(0.2-0.4)$ & $0.38(0.2-0.6)$ & $0.65(0.5-0.8)$ \\
\hline PPV (95\% Cl) & 79.0 (73.4-83.7) & $57.4(53.9-60.8)$ & 70.0 (62.3-76.7) & $41.4(36.5-46.6)$ & $33.3(29.4-37.5)$ & $34.9(28.5-41.8)$ \\
\hline NPV $(95 \% \mathrm{Cl})$ & $81.8(76.2-86.3)$ & $72.5(63.8-79.8)$ & $62.1(58.0-66.0)$ & 92.8 (88.7-95.5) & $90.2(85.3-93.6)$ & $84.4(80.7-87.6)$ \\
\hline AUC (95\% CI) & $0.86(0.82-0.90)$ & $0.61(0.55-0.66)$ & $0.67(0.62-0.72)$ & $0.78(0.73-0.82)$ & $0.68(0.63-0.73)$ & $0.64(0.58-0.69)$ \\
\hline$P$ value & $<0.001$ & 0.001 & $<0.001$ & $<0.001$ & $<0.001$ & $<0.001$ \\
\hline
\end{tabular}

PPV, positive predictive value; NPV, negative predictive value; PLR, positive likelihood ratio; NLR, negative likelihood ratio; WBC, white blood cell; CRP, C-reactive protein; UNGAL, urinary neutrophil gelatinase-associated lipocalin; UTI, urinary tract infection; APN, acute pyelonephritis; AUC, areas under the curve; $\mathrm{Cl}$, confidence interval.

Boldface indicates a statistically significant difference with $P<0.05$. 
UTI and APN, the AUCs for UNGAL level were significantly higher than that for the CRP level and WBC counts, respectively. The PPV, NPV, PLR, and NLR of UNGAL were superior to those of the serum CRP and WBC for detecting UTI and APN at each cutofflevel.

The NGAL is an iron-carrier protein derived from human neutrophils. It plays a key role in the innate immune response in case of bacterial infections. ${ }^{25}$ ) Previous studies have uniformly shown that the UNGAL levels are significantly elevated in response to UTIs, especially in gram-negative bacterial infections. ${ }^{9,25,26)}$ Animal studies comparing rats injected with Escherichia coli and with saline showed that the UNGAL levels were upregulated in the experimental UTI and APN model. ${ }^{15,27)}$ In a clinical study, Yilmaz et al. ${ }^{16}$ ) reported that the uNGAL level and the uNGAL/creatinine ratio were higher in the UTI group than in the control group. We also previously reported that the uNGAL and uNGAL/creatinine ratio were useful in predicting UTI and APN in children. ${ }^{28)}$ The current study confirmed that uNGAL might be helpful for identifying UTI and APN in children with fever. The uNGAL level was higher in the UTI group than in the non-UTI group. Among children with UTIs, uNAGL was more elevated in patients with APN, compared with those without APN. Given that uNGAL is a specific indicator of damaged genitourinary epithelium, ${ }^{16,17)}$ increased uNGAL may suggest renal tubular injury as well as UTI. The commercial kits we used measures the monomeric form of NGAL in urine predominantly. While urinary monomeric NGAL is primarily of renal origin, it can be released by urinary neutrophils in UTI states. ${ }^{29)}$ In the present study we excluded the patients with previous UTIs, AKI, CKD, and congenital urinary tract anomalies, however, subclinical renal tubular injury might affect the levels of uNGAL. Nevertheless, the serum creatinine level was higher in the non-UTI group, compared with the UTI group, and it was not even different between the non-APN and APN groups. Therefore, changes in UNGAL concentration are thought to be independent of serum creatinine level, and the difference in creatinine levels between the 2 groups may represent age-related variation. ${ }^{30)}$ Meanwhile, fever duration was shorter in the UTI group, compared with the non-UTI group. It is plausible that the prompt treatment with empiric antibiotics could shorten the duration of fever in the UTI group although the exact cause remains unclear.

It appears that uNGAL is superior to other biomarkers such as PCT, CRP, and WBC in distinguishing between acute bacterial and viral infections. ${ }^{31)}$ In a total of 155 patients with acute infections, serum measurement of NGAL was more useful in differentiating bacterial infections from viral ones, compared with the CRP and WBCs. ${ }^{32}$ Although leukocytosis, neutrophilia, and the elevation of the CRP are common findings in UTI, these may be nonspecific markers of inflammation as seen above. In this study, we identified that the uNGAL level could be useful in distinguishing UTI from viral infections in children with fever. While the serum CRP and WBC concentrations were all elevated in patients with UTI, they were not associated with the presence of UTI independently. Young age and increased UNGAL level independently predicted the presence of UTI in a multivariable logistic model. Even in children with UTIs, only the uNGAL was also independently associated with more severe infections, or APN. While the univariable analyses showed that the serum WBC and CRP were related with the presence of APN, they were not independent predictors of APN in a multivariable analysis. Notably, we previously showed that only pNGAL level was an independent predictor of APN in children with febrile UTI when female gender, VUR, pNGAL, and serum PCT, WBC, and CRP were included in a multivariable logistic regression model. ${ }^{20)}$ These findings suggest that $\mathrm{pNGAL}$ and uNGAL could be useful in detecting UTI and APN and be more specific than the serum CRP and WBC in predicting UTI and APN in children with fever.

In the ROC analyses, we also found that uNGAL is the most reliable biomarker in detecting UTI and APN than other examined biomarkers. For predicting UTI and APN, the AUC for uNGAL level was significantly higher than that for the CRP level and WBC counts, respectively. PPV, NPV, PLR, and NLR of uNGAL were superior to those of the serum CRP and WBC measurement for detecting UTI and APN at each cutoff level. Yilmaz et al. ${ }^{16)}$ noted that the cutoff level for the uNGAL in predicting UTI was $20 \mathrm{ng} / \mathrm{mL}$ in 60 patients with symptomatic UTI and 29 healthy controls. The sensitivity was $97 \%$, and specificity was $76 \%$. In our previous study, ${ }^{28)}$ the best cutoff values of the uNGAL were $23.95 \mathrm{ng} / \mathrm{mL}$ (sensitivity, 82.4\%; specificity, 83.6\%) for UTI and $73 \mathrm{ng} / \mathrm{mL}$ (sensitivity, 75\%; specificity, 73.7\%) for APN. The uNGAL cutoff value was somewhat different to that of this study, which is $36.5 \mathrm{ng} /$ $\mathrm{mL}$ for UTI and $53.1 \mathrm{ng} / \mathrm{mL}$ for APN. This may be because of the difference of the control groups. In the previous study, the control group comprised healthy children with no evidence of infection or fever while in this study the control group comprised children with viral infections or fever. Consistent with our findings, among 260 febrile infants and children younger than 24 months, Lubell et al. ${ }^{10)}$ also identified that the optimal cutoff of UNGAL for UTI was $39.1 \mathrm{ng} / \mathrm{mL}$, with an AUC of 0.978, and a sensitivity of $97.1 \%$ and a specificity of $95.6 \%$. Compared with UA, uNGAL showed higher overall diagnostic accuracy for UTI with greater sensitivity and similar specificity. As another study with opposite results, Krzemien et al. ${ }^{33)}$ showed the uNGAL was not specific for detecting APN in children with febrile UTIs. They investigated if uNGAL and kidney injury molecule-1 (uKIM-1) could be a useful biomarker to predict APN in 54 children age 1-24 months with first febrile UTI. In their study, the uNGAL, uKIM-1, PCT, CRP, and the serum WBC measurements were performed together. They showed that only the PCT and CRP were good diagnostic factors of APN. The AUC for the PCT was 0.894, and that for the CRP was 0.719. Interestingly, the uNGAL and uKIM-1 did not have predictive value for identifying APN in febrile UTI in their study. The small sample size may have resulted in these conflicting results, and further prospective studies with a larger number of patients are needed. In 
our previous study comparing the diagnostic accuracy of the pNGAL to the PCT, CRP, and WBC for APN prediction, ${ }^{20)}$ the pNGAL had the highest AUC value of 0.893 , followed by the CRP, PCT, and WBC. The sensitivity (86\%) and specificity (85\%) measured as part of the ROC analysis were the highest for the pNGAL. The pNGAL showed the highest PPV (81\%) and NPV (89\%) in predicting the APN among all biomarkers tested. In the present study, uNGAL was also more useful for predicting UTI and APN, compared with serum CRP and WBC. Serum CRP and WBC were neither sensitive nor specific for detecting UTI. ${ }^{34)}$ These findings suggest that uNGAL as well as pNGAL could be more accurate than serum CRP and WBC for predicting UTI or APN in children. The NGAL measurement in blood and urine would be helpful in diagnosing UTI and APN in children with fever. Given that the reliable diagnosis of UTI is often difficult and imaging studies for diagnosing APN are widely used in children, the NGAL measurement would be an important adjunct in establishing the diagnosis and management of UTI in children with fever.

There were limitations in this study. First, it was retrospective, and there was a relatively small population from only one center. Second, the uNGAL level was not corrected with urine creatinine. Finally, studies of the association between biomarkers and renal scarring were not performed because of the short study period and the follow-up loss. Results must be validated in a larger, multicenter, prospective study of various children from age groups over a longer period.

In conclusion, the uNGAL may serve as a useful biomarker in detecting UTI and APN in children with fever. The diagnostic accuracy of the uNGAL for UTI and APN may be higher than the serum CRP and WBC in children with fever. The NGAL measurement can clarify the results of UA or urine culture in children with suspected UTI and obviate the need to perform extensive radiological evaluation for APN in children with febrile UTIs. Further investigations are required in a larger population for diagnosing and managing febrile UTIs and APN in children.

\section{Conflicts of interest}

No potential conflict of interest relevant to this article was reported.

\section{References}

1. Shaikh N, Morone NE, Bost JE, Farrell MH. Prevalence of urinary tract infection in childhood: a meta-analysis. Pediatr Infect Dis J 2008;27:3028

2. Chaudhari PP, Monuteaux MC, Bachur RG. Urine concentration and pyuria for identifying UTI in infants. Pediatrics 2016;138:e20162370.

3. Becknell B, Schober M, Korbel L, Spencer JD. The diagnosis, evaluation and treatment of acute and recurrent pediatric urinary tract infections. Expert Rev Anti Infect Ther 2015;13:81-90.

4. Montini G, Tullus K, Hewitt I. Febrile urinary tract infections in children. NEngl J Med 2011;365:239-50.

5. Park YS. Renal scar formation after urinary tract infection in children. Korean J Pediatr 2012;55:367-70.

6. Williams GJ, Macaskill P, Chan SF, Turner RM, Hodson E, Craig JC.
Absolute and relative accuracy of rapid urine tests for urinary tract infection in children: a meta-analysis. Lancet Infect Dis 2010;10:240-50

7. Bachur R, Harper MB. Reliability of the urinalysis for predicting urinary tract infections in young febrile children. Arch Pediatr Adolesc Med 2001;155:60-5.

8. Mishra J, Ma Q, Prada A, Mitsnefes M, Zahedi K, Yang J, et al. Identification of neutrophil gelatinase-associated lipocalin as a novel early urinary biomarker for ischemic renal injury. J Am Soc Nephrol 2003; 14:2534-43.

9. Paragas N, Kulkarni R, Werth M, Schmidt-Ott KM, Forster C, Deng R, et al. Alpha-Intercalated cells defend the urinary system from bacterial infection. J Clin Invest 2014;124:2963-76.

10. Lubell TR, Barasch JM, Xu K, Ieni M, Cabrera KI, Dayan PS. Urinary neutrophil gelatinase-associated lipocalin for the diagnosis of urinary tract infections. Pediatrics 2017;140:e20171090.

11. Cowland JB, Borregaard N. Molecular characterization and pattern of tissue expression of the gene for neutrophil gelatinase-associated lipocalin from humans. Genomics 1997;45:17-23.

12. Zappitelli M, Washburn KK, Arikan AA, Loftis L, Ma Q, Devarajan P, et al. Urine neutrophil gelatinase-associated lipocalin is an early marker of acute kidney injury in critically ill children: a prospective cohort study. Crit Care 2007;11:R84.

13. Forster CS, Devarajan P. Neutrophil gelatinase-associated lipocalin: utility in urologic conditions. Pediatr Nephrol 2017;32:377-81.

14. Berger T, Togawa A, Duncan GS, Elia AJ, You-Ten A, Wakeham A, et al. Lipocalin 2-deficient mice exhibit increased sensitivity to Escherichia coli infection but not to ischemia-reperfusion injury. Proc Natl Acad Sci U S A 2006;103:1834-9.

15. Ichino M, Kuroyanagi Y, Kusaka M, Mori T, Ishikawa K, Shiroki R, et al. Increased urinary neutrophil gelatinase associated lipocalin levels in a rat model of upper urinary tract infection. J Urol 2009;181:2326-31.

16. Yilmaz A, Sevketoglu E, Gedikbasi A, Karyagar S, Kiyak A, Mulazimoglu $\mathrm{M}$, et al. Early prediction of urinary tract infection with urinary neutrophil gelatinase associated lipocalin. Pediatr Nephrol 2009;24:2387-92.

17. Abassi Z, Sagi O, Armaly Z, Bishara B. Neutrophil gelatinase-associated lipocalin (NAGL): a novel biomarker for acute kidney injury. Harefuah 2011;150:111-6, 207, 206.

18. Jagadesan I, Agarwal I, Chaturvedi S, Jose A, Sahni RD, Fleming JJ. Urinary neutrophil gelatinase associated lipocalin - a sensitive marker for urinary tract infection in children. Indian J Nephrol 2019;29:340-4.

19. Nickavar A, Safaeian B, Valavi E, Moradpour F. Validity of neutrophil gelatinase associated lipocaline as a biomarker for diagnosis of children with acute pyelonephritis. Urol J 2016;13:2860-3.

20. Kim BK, Yim HE, Yoo KH. Plasma neutrophil gelatinase-associated lipocalin: a marker of acute pyelonephritis in children. Pediatr Nephrol 2017;32:477-84.

21. Hong IK, Chung MH, Bin JH, Lee KY, Kim JS, Kim CH, et al. Prediction of vesicoureteral reflux in children with febrile urinary tract infection using relative uptake and cortical defect in DMSA scan. Pediatr Neonatol 2018;59:618-23.

22. Zhang X, Xu H, Zhao RF. Efficacy of acute (99m)Tc-dimercaptosuccinic acid scan in predicting vesicoureteral reflux among young children with febrile urinary tract infection. Zhonghua Er Ke Za Zhi 2010;48:334-7.

23. Keays MA, Guerra LA, Mihill J, Raju G, Al-Asheeri N, Geier P, et al. Reliability assessment of Society for Fetal Urology ultrasound grading system for hydronephrosis. J Urol 2008;180(4 Suppl):1680-2; discussion 2-3.

24. Hanley JA, McNeil BJ. The meaning and use of the area under a receiver operating characteristic (ROC) curve. Radiology 1982;143:29-36.

25. Flo TH, Smith KD, Sato S, Rodriguez DJ, Holmes MA, Strong RK, et al. Lipocalin 2 mediates an innate immune response to bacterial infection by sequestrating iron. Nature 2004;432:917-21.

26. Czaja CA, Stamm WE, Stapleton AE, Roberts PL, Hawn TR, Scholes $\mathrm{D}$, et al. Prospective cohort study of microbial and inflammatory events immediately preceding Escherichia coli recurrent urinary tract infection in women. J Infect Dis 2009;200:528-36.

27. Lee HE, Lee SH, Baek M, Choi H, Park K. Urinary measurement of 
neutrophil gelatinase associated lipocalin and kidney injury molecule-1 helps diagnose acute pyelonephritis in a preclinical model. J Biomark 2013;2013:413853.

28. Yim HE, Yim H, Bae ES, Woo SU, Yoo KH. Predictive value of urinary and serum biomarkers in young children with febrile urinary tract infections. Pediatr Nephrol 2014;29:2181-9.

29. Glassford NJ, Schneider AG, Xu S, Eastwood GM, Young H, Peck L, et al. The nature and discriminatory value of urinary neutrophil gelatinaseassociated lipocalin in critically ill patients at risk of acute kidney injury. Intensive Care Med 2013;39:1714-24

30. Swedko PJ, Clark HD, Paramsothy K, Akbari A. Serum creatinine is an inadequate screening test for renal failure in elderly patients. Arch Intern Med 2003;163:356-60.

31. Venge P, Douhan-Hakansson L, Garwicz D, Peterson C, Xu S, Pauksen K. Human neutrophil lipocalin as a superior diagnostic means to distinguish between acute bacterial and viral infections. Clin Vaccine Immunol 2015; 22:1025-32.

32. Yu Z, Jing $\mathrm{H}$, Hongtao P, Furong J, Yuting J, Xu S, et al. Distinction between bacterial and viral infections by serum measurement of human neutrophil lipocalin (HNL) and the impact of antibody selection. J Immunol Methods 2016;432:82-6.

33. Krzemien G, Panczyk-Tomaszewska M, Kotula I, Demkow U, Szmigielska A. Diagnostic accuracy of urine neutrophil gelatinase-associated lipocalin and urine kidney injury molecule- 1 as predictors of acute pyelonephritis in young children with febrile urinary tract infection. Cent Eur J Immunol 2019;44:174-80.

34. Ayazi P, Mahyar A, Daneshi MM, Jahani Hashemi H, Pirouzi M, Esmailzadehha N. Diagnostic accuracy of the quantitative c-reactive protein, erythrocyte sedimentation rate and white blood cell count in urinary tract infections among infants and children. Malays J Med Sci 2013;20:40-6.

How to cite this article: Moon JH, Yoo KH, Y HE. Urinary neutrophil gelatinase-associated lipocalin: a marker of urinary tract infection among febrile children. Clin Exp Pediatr 2021; 64:347-54. https://doi.org/10.3345/cep.2020.01130 